\title{
Impact of COVID-19 lockdown on flash and real-time glucose sensor users with type 1 diabetes in England
}

\author{
Joshi Prabhu Navis ${ }^{1} \cdot$ Lalantha Leelarathna $^{1,2} \cdot$ Womba Mubita $^{1} \cdot$ Andrea Urwin $^{1} \cdot$ Martin K. Rutter $^{1,2}$. \\ Jonathan Schofield ${ }^{1} \cdot$ Hood Thabit ${ }^{1,2}$ (1)
}

Received: 10 August 2020 / Accepted: 1 October 2020 / Published online: 16 October 2020

(c) The Author(s) 2020

\begin{abstract}
Aims People with type 1 diabetes (T1D) face the daily task of implementing self-management strategies to achieve their glycaemic goals. The UK COVID-19 lockdown has had an impact on day-to-day behaviour, which may affect diabetes selfmanagement and outcomes. We assessed whether sensor-based outcomes pre- and during lockdown periods were different in a cohort of glucose sensor users with T1D.

Methods Data were collected from Freestyle Libre (FSL) or Dexcom G6 sensor users who remotely shared their data with the diabetes clinic web platform. Sensor metrics according to international consensus were analysed and compared between pre-lockdown period and 2 and 3 weeks into lockdown (periods 1 and 2).

Results Two hundred and sixty-nine T1D patients (baseline HbA1c $57 \pm 14 \mathrm{mmol} / \mathrm{mol}$ ) were identified as FSL $(n=190)$ or Dexcom G6 $(n=79)$ users. In patients with sensor use $>70 \%(N=223)$, compared to pre-lockdown period percentage TIR $3.9-10 \mathrm{mM}$ (TIR) significantly increased during period 1 (59.6 \pm 18.2 vs. $57.5 \pm 17.2 \%, p=0.002)$ and period $2(59.3 \pm 18.3$ vs. $57.5 \pm 17.2 \%, p=0.035$ ). The proportion of patients achieving TIR $\geq 70 \%$ increased from $23.3 \%$ pre-lockdown to $27.8 \%$ in period 1 and $30.5 \%$ in period 2. A higher proportion also achieved the recommended time below and above range, and coefficient of variation in periods 1 and 2 . Dexcom G6 users had significantly lower $\%$ time below range $(<3.9 \mathrm{mM})$ compared to FSL users during both lockdown periods (period 1: Dexcom G6 vs. FSL: 1.8\% vs. 4\%; period 2: 1.4\% vs. 4\%, $p<0.005$ for both periods).

Conclusion Sensor-based glycaemic outcomes in people with T1D in the current cohort improved during COVID-19 lockdown, which may be associated with positive changes in self-management strategies. Further work is required to evaluate long-term sustainability and support.
\end{abstract}

Keywords COVID-19 lockdown $\cdot$ Flash glucose monitoring $\cdot$ Real-time continuous glucose monitoring $\cdot$ Type 1 diabetes

Managed by Antonio Secchi.

Joshi Prabhu Navis, Lalantha Leelarathna: Joint first author

Hood Thabit

hood.thabit@mft.nhs.uk

1 Diabetes, Endocrinology and Metabolism Centre, Manchester University NHS Foundation Trust, Manchester Academic Health Science Centre, Manchester M13 9WL, UK

2 Division of Diabetes, Endocrinology and Gastroenterology, Faculty of Biology, Medicine and Health, University of Manchester, Manchester, M13 9WL, UK

\section{Introduction}

One of the self-management goals in type 1 diabetes is achieving and maintaining optimal glycaemic control to reduce the risk of complications [1]. This entails the complex daily tasks of frequent monitoring of glucose levels, bolusing insulin at specific times and varying insulin doses according to meal size, physical activities and health status, whilst taking precautionary measures to avoid significant dysglycaemia $[2,3]$. The burden of these tasks is compounded by the demands of daily living, causing many people with type 1 diabetes to struggle and experience burnout leading to poor glycaemic outcomes [4-6].

On 23 March 2020, the UK Government implemented movement restriction or lockdown measures in response to 
the COVID-19 virus global pandemic [7] through the Coronavirus Act, which allowed the UK police to enforce the lockdown [8]. Members of the public, including people with diabetes, were advised to stay at home except for certain "very limited purposes", such as shopping for basic necessities or for any medical need. Routine outpatient activities were suspended during lockdown as hospitals and healthcare professionals across the UK responded to the pandemic. The lockdown measure has had a unique and widespread impact on societal behaviour, which may have directly or indirectly affected diabetes self-management.

Glucose monitoring technologies, such as flash and continuous glucose systems, are used in routine clinical practice and provide access to real-time glucose values and predictive glucose trends. They also enable remote sharing of glycaemic data between patients and their healthcare professionals through cloud-based web platforms. The benefits of flash and continuous glucose monitoring have been widely published [9-12]. However, their role in evaluating the glycaemic impact of the COVID-19 lockdown measure in the UK is not widely known and may have implications for future diabetes service development.

The objective of this service evaluation was to analyse and compare glycaemic outcomes before and during lockdown in a cohort of people with diabetes using flash and continuous glucose monitors.

\section{Methods}

This was a retrospective, observational, single-centre, service evaluation in a large teaching hospital in the UK. As an audit and service evaluation, no ethical approval was required. Data were collected from outpatient electronic records and glucose monitoring web-based platforms (Libreview; Abbott diabetes care and Dexcom clarity; Dexcom Inc). Patients had provided online informed consent for their data to be remotely connected to and shared with the diabetes clinic.

Sensor data from Libreview and Dexcom clarity were analysed for each of the following periods: pre-lockdown (1-14 February 2020), early lockdown (period 1; 1-14 April 2020) and mid-lockdown (period 2; 1-14 May 2020). Patients were eligible for inclusion if they met the following criteria: history of diabetes, on multiple daily injections (MDI) or insulin pump therapy and using Freestyle Libre flash glucose monitoring (FSL) or Dexcom G6 continuous glucose monitoring on their smartphone device. Analysis was performed in those with at least $70 \%$ sensor data available during all 3 periods (pre-, early- and mid-lockdown).

In keeping with international consensus on CGM reporting guidelines [13], we analysed the following glycaemic metrics of each patient using the proprietary reporting function of Libreview and Dexcom Clarity software: \% time in glucose range (3.9-10.0 mmol/l) (TIR), \% time below range $(<3.9 \mathrm{mmol} / \mathrm{l}), \%$ time above range $(>10.0 \mathrm{mmol} / \mathrm{l})$, coefficient of variation $(\mathrm{CV} \%)$ and estimated $\mathrm{HbA} 1 \mathrm{c}$ value. Estimated $\mathrm{HbA} 1 \mathrm{c}(\mathrm{eA} 1 \mathrm{c})$ values were obtained from each patient's Libreview and Dexcom clarity account, calculated using a validated equation [14]. Adherence to sensor use was evaluated by assessing $\%$ of sensor data availability for both FSL and continuous glucose monitors, and number of daily scans for FSL users.

Data on age, gender, postcode, diabetes duration and type of insulin delivery were collected from outpatient electronic records. Participants were assigned an English index of multiple deprivation rank (IMD, https://www.gov.uk/gover nment/statistics/english-indices-of-deprivation-2019) based upon their postcode. These were then grouped by deprivation decile defined by their position in the ranks from the 32,844 small areas in England subdivided into 10 equal groups, with 1 being the most socio-economically deprived and 10 being the least deprived.

\section{Statistical analysis}

Analyses were performed using paired and unpaired sample $t$ tests for normal variable distributions, or Wilcoxon signed rank tests for non-normal variable distributions. The change in TIR for different glucose monitoring systems and insulin delivery modality was adjusted by including baseline TIR as a covariate in the analysis of covariance models. Correlation analysis was performed between laboratory $\mathrm{HbA} 1 \mathrm{c}$ and eA1c, TIR, age, duration of diabetes and index of multiple deprivation. Values were presented as mean \pm SD or median (interquartile range). The hypothesis testing was ordered at the 0.05 level without any control for multiple testing. We completed analyses with SPSS (IBM software, Hampshire $\mathrm{UK}$, version 25). All $p$ values are two-sided.

\section{Results}

Two hundred and sixty-nine type 1 diabetes patients were identified as using FSL $(n=190)$ or Dexcom G6 $(n=79)$ on smartphone devices. Table 1 summarises their baseline characteristics. The majority were using an insulin pump (70\%) as their method of insulin delivery. Mean laboratory HbA1c before lockdown announcement was $57 \mathrm{mmol} / \mathrm{mol}$ (7.3\%).

\section{Pre-lockdown sensor analysis $(n=269)$}

Pre-lockdown mean \pm SD TIR was $56.0 \pm 18.0 \%$, time above range was $39.1 \pm 19.9 \%$, and median (IQR) time below range was 3\% IQR 1-6. Twenty-three percent of patients achieved TIR $\geq 70 \%$. Glucose variability pre-lockdown, as measured 
Table 1 Baseline characteristics

\begin{tabular}{ll}
\hline Characteristics & Data \\
\hline Sex, $N(\%)$ & \\
Female & $123(46)$ \\
Male & $146(54)$ \\
Age(years) & $41.4 \pm 12.9$ \\
Duration of diabetes(years) & $23.6 \pm 12.9$ \\
HbA1c mmol/mol & $57 \pm 14$ \\
HbA1c \% & $7.3 \pm 1.3$ \\
HbA1c \% subgroups & \\
Multiple daily injections & $7.3 \pm 1.2$ \\
Insulin pump therapy & $7.4 \pm 1.3$ \\
Freestyle Libre & $7.3 \pm 1.3$ \\
Dexcom G6 & $7.4 \pm 1.3$ \\
Insulin delivery method, $N(\%)$ & \\
Multiple daily injections & $80(30)$ \\
Insulin pump therapy & $189(70)$ \\
Glucose monitoring system & \\
Freestyle Libre & $190(71)$ \\
Dexcom G6 & $79(29)$ \\
Index of multiple deprivation rank, median & $15,369(8203-25,132)$ \\
(IQR) & \\
Index of multiple deprivation decile, median & $5(3-8)$ \\
(IQR) & \\
\hline
\end{tabular}

Data are mean $\pm \mathrm{SD}$, unless specified otherwise. $N=269$

by sensor glucose coefficient of variation, was $37.3 \pm 6.4 \%$. A significant correlation was shown between pre-lockdown laboratory-based $\mathrm{HbA} 1 \mathrm{c}$ and sensor-based estimated $\mathrm{HbA} 1 \mathrm{c}$ $(r=0.67, p<0.005)$.

There were no differences in $\%$ TIR, time above or below range between MDI and insulin pump users. Pre-lockdown $\%$ TIR for insulin pump vs. MDI was $56.5 \pm 17.5$ vs. $55.0 \pm 19.1 \%, p=0.56$; time above range was $38.5 \pm 19.7$ vs. $40.5 \pm 20.5 \%, p=0.46$; and median (IQR) time below range was 3.0(1.0-6.0) vs. 3.8(1.0-6.0)\%, $p=0.47$. Median (IQR) sensor use pre-lockdown was high, 97 (91-99) \%. No significant difference was observed in the \% TIR $(55.8 \pm 17.2$ vs. $56.6 \pm 19.9 \% p=0.74)$ and time above range $(38.6 \pm 19.2$ vs. $40.4 \pm 21.6 \%, p=0.49)$ pre-lockdown between FSL and Dexcom G6 users; however, \% median (IQR) time below range was significantly lower in Dexcom G6 users (FSL: $4.0(2-7) \%$ vs. Dexcom: $1.6(0.5-3.6) \%, p<0.005)$. Among FSL users, the median (IQR) number of scans was 10 (7-14) per day.

\section{Sensor data comparison between pre-lockdown and lockdown periods $(N=223)$}

Two hundred and twenty-three patients $(\mathrm{FSL}=152$, Dexcom G6 $=71$ ) had at least $70 \%$ sensor data available during all 3 periods (pre-lockdown, periods 1 and 2; Table 2). The $\%$ TIR (3.9-10.0 mmol/l) during lockdown (periods 1 and 2) was significantly higher compared to pre-lockdown $(59.6 \pm 18.2$ vs. $57.5 \pm 17.2 \%, p=0.002$ and $59.3 \pm 18.3$ vs. $57.5 \pm 17.2 \%, p=0.035$, respectively). Hyperglycaemia as measured by time above range was significantly lower in period 1 compared to pre-lockdown (period 1: $35.3 \pm 20.2 \%$ vs. pre-lockdown: $37.9 \pm 18.9 \%, p=0.001)$, but was similar to baseline in period $2(p=0.098)$. Conversely, glycaemic variability as measured by sensor glucose coefficient of variation was lower during period 2 than pre-lockdown (period 2: $36.0 \pm 6.4 \%$ vs. pre-lockdown: $37.0 \pm 5.8 \%, p=0.003$ ) but similar during period $1(p=0.24)$.

No significant change for $\%$ time spent below range during both lockdown periods was observed in the whole cohort. Those using the Dexcom G6 CGM, however, had a significantly lower \% time below range compared to FSL users during both lockdown periods (Dexcom G6 vs. FSL, period 1 median (IQR): $1.8(0.4-4.8)$ vs. 4 IQR (2-9)\%. Period 2 median (IQR): $1.4(0.3-4.4)$ vs. $4(2-8) \% . p<0.005$ for both periods).

The proportion of patients achieving clinically recommended $\geq 70 \%$ TIR [13] increased from pre-lockdown to periods 1 and 2 (see Table 3). Similarly, the proportion of patients achieving the clinically recommended time below range, above range and coefficient of variation in Table 3 also increased from pre-lockdown to period 2. Thirty-five percent of patients had clinically significant improvement in TIR (change $\geq 5 \%$ ) during period 2 compared to

Table 2 Sensor-based glycaemic outcomes (paired analysis of the same patient across the 3 time periods)

\begin{tabular}{llllll}
\hline & Pre-lockdown & Period 1 & Period 2 & $\begin{array}{l}p \text {-value } \\
\text { pre-lockdown vs. } \\
\text { period 1 }\end{array}$ & $\begin{array}{l}p \text {-value } \\
\text { pre-lockdown } \\
\text { vs. period 2 }\end{array}$ \\
\hline \% Time in range & $57.5 \pm 17.2$ & $59.6 \pm 18.2$ & $59.3 \pm 18.3$ & 0.002 & 0.035 \\
$(3.9-10.0$ mmol/1) & & & & & \\
\% Time below range $(<3.9 \mathrm{mmol} / \mathrm{l})$ & $3.0(1-6)$ & $4.0(1-7)$ & $3.0(1-6)$ & 0.056 & 0.85 \\
$\%$ Time above range $(>10.0 \mathrm{mmol} / 1$ & $37.9 \pm 18.9$ & $35.3 \pm 20.2$ & $36.4 \pm 20.0$ & 0.001 & 0.098 \\
Coefficient of variation $(\%)$ & $37.0 \pm 5.8$ & $36.6 \pm 6.8$ & $36.0 \pm 6.4$ & 0.24 & 0.003 \\
\hline
\end{tabular}

Data are mean \pm SD or median IQR. $N=223$ 
Table 3 Proportion of patients achieving recommended sensorbased glycaemic targets

\begin{tabular}{llll}
\hline & Pre-lockdown & Period 1 & Period 2 \\
\hline \% Achieving $\geq 70 \%$ time in range & 23.3 & 27.8 & 30.5 \\
\% Achieving $\leq 4 \%$ time below range & 61.9 & 57.4 & 62.3 \\
\% Achieving $\leq 25 \%$ time above range & 26.5 & 34.5 & 33.2 \\
\% Achieving $\leq 36 \%$ coefficient of variation & 42.2 & 43.9 & 48.9 \\
\hline
\end{tabular}

Data are $\% . N=223$ pre-lockdown, whilst $38 \%$ had clinically significant improvement in estimated $\mathrm{HbA} 1 \mathrm{c}$ (change $\geq 0.3 \%$ ). Conversely, there was a deterioration of TIR (change $\geq 5 \%$ ) in $26 \%$ and estimated $\mathrm{HbA} 1 \mathrm{c}$ (change $\geq 0.3 \%$ ) in $23 \%$ of patients.

\section{Factors associated with improvement and deterioration of TIR.}

Table 4 summarises the characteristics of patients whose $\%$ TIR improved by $\geq 5 \%(n=91)$ or deteriorated by $\geq 5 \%$ $(n=71)$ between pre-lockdown and period 2. There was no difference in age, duration of diabetes or index of multiple deprivation rank and deciles between those with improved vs. deteriorated TIR. The improvement in TIR between period 2 and pre-lockdown was negatively associated with baseline pre-lockdown TIR $(r=-0.3, p<0.005)$ indicating greater improvement in TIR for those with lower TIR at baseline. In keeping with this observation, baseline HbA1c was higher in patients whose $\%$ TIR improved by $5 \%$ or more.

There were no differences in \% TIR for periods 1 and 2, between FSL and Dexcom G6 or between insulin pump users and MDI. The \% TIR for Dexcom G6 vs. Libre in period 1 was $56 \pm 20$ vs. $61 \pm 16 \%, p=0.06$, while for period 2 was $57 \pm 21$ vs. $60 \pm 17 \%, p=0.14$. The $\%$ TIR for insulin pump users vs. MDI in period 1 was $60 \pm 17$ vs. $59 \pm 20 \%$, $p=0.9$, and period 2 was $59 \pm 18$ vs. $59 \pm 18 \%, p=0.97$. Adjusting for baseline \% TIR, the change in TIR between pre-lockdown and period 2 was not significantly different for both glucose monitoring systems (FSL and Dexcom G6, $p=0.22$ ) and insulin delivery modalities (MDI and insulin pump, $p=0.49$ ). No significant correlation was found between absolute TIR in period 1 and period 2, with age, duration of diabetes or index of multiple deprivation rank and deciles.

\section{Discussion}

In this study, we describe the changes that occurred in sensor-based metrics in a cohort of adults with type 1 diabetes who shared their FSL or Dexcom G6 sensor data with our clinical service. We showed that compared to pre-lockdown period, a significant increase in proportion of time spent in range was observed in both lockdown periods accompanied by an increase in the proportion of patients achieving the recommended TIR of $\geq 70 \%$. Over $40 \%$ of the cohort had clinically meaningful improvements in both TIR and estimated HbA1c during the lockdown period. Other sensor-based outcomes such as time below range, above range and glycaemic variability were comparable or significantly improved during lockdown period in this cohort.

The global COVID-19 pandemic has placed significant challenges on healthcare systems across the world. Similar to our centre, many healthcare institutions had to suspend usual service provisions such as outpatient clinic

Table 4 Comparison of clinical characteristics of patients with better vs. worse glycaemic control in period 2 compared to pre-lockdown

\begin{tabular}{lll}
\hline Characteristics & $\begin{array}{l}\text { Time in range change (period 2-pre- } \\
\text { lockdown) increased by } \geq 5 \%\end{array}$ & $\begin{array}{l}\text { Time in range change (period 2-pre- } \\
\text { lockdown) decreased by } \geq 5 \%\end{array}$ \\
\hline Sex, $\mathrm{n}(\%)$ & & $33(27)$ \\
Female & $48(39)$ & $38(26)$ \\
Male & $51(35)$ & $41.6 \pm 11.9$ \\
Age & $41.0 \pm 13.2$ & $24.3 \pm 13.9$ \\
Duration of diabetes & $23.9 \pm 13.3$ & $60.8 \pm 15.6$ \\
Time in range, pre-lockdown (\%) & $48.9 \pm 16.9$ & $57.6 \pm 11.4$ \\
Estimated HbAlc mmol/mol, pre-lockdown & $65.3 \pm 16.5$ & $17,687(9141-25,748)$ \\
Index of multiple deprivation rank, median (IQR) & $14,155(6368-25,626)$ & $6(3-8)$
\end{tabular}

Data are $\mathrm{N}(\%)$ or mean \pm SD unless specified otherwise 
appointments to manage the COVID-19 pandemic and had their healthcare workers such as diabetes specialists and educators redeployed to the frontline. A report from Australia showed that reduced access to primary care and hospital services for diabetes, combined with fear of exposure to the virus, had led to a significant drop in access to usual diabetes care [15]. The authors, however, suggested that this risk was mitigated by implementing proactive measures, which included improving access to technologies such as glucose monitoring systems. Use of telemedicine in diabetes care during this pandemic has also been increasing throughout the world including in our own institution, which at present has replaced most face-to-face contacts to minimise exposure and to adhere to social distancing rules. A recent study from India showed that glycaemic control in people with type 1 diabetes worsened during lockdown period mainly due to non-availability of insulin and glucose testing kits [16]. This suggests that different countries with different healthcare systems and resources cope differently in the circumstances.

Other studies from other countries have shown comparable results to ours, suggesting that COVID-19 lockdown measures have not had a deleterious effect on short-term glycaemic control in people with T1D. A study in the north eastern region of Italy involving 33 FSL users with type 1 diabetes analysed sensor-based outcomes during the first 7 days of lockdown [17]. The authors also performed a subanalysis in a smaller cohort $(N=13)$ who continued working during lockdown. In those who stayed at home, mean and standard deviation sensor glucose, TIR and time above range all improved compared to the weeks before lockdown. However, no changes in glycaemic outcomes were observed in those who continued working. The authors thus speculated that the improvements observed in the former may have likely been due to having more time to focus on diabetes self-management, such as timing of boluses and estimating meal composition, while not being exposed to the demands and stress at the workplace. A Spanish study evaluating 17 Dexcom G5 and 75 FSL users reported significant reductions in TIR, time above range and glucose management indicator (GMI), whilst time below range was unchanged [18]. Dover and colleagues recently analysed paired data from 572 FSL users in Scotland and similarly found improvements in TIR and estimated HbA1c [19]. The authors indicated that socio-economic deprivation was an independent predictor of glycaemic control decline during lockdown.

Our findings advance the observations from the aforementioned Italian and Spanish studies by having a larger sample size, including those on MDI and insulin pump therapy, and comparing 3 time points (pre-lockdown, early and mid-lockdown). Compared to the study from Dover and colleagues, in addition to FSL our analyses also included real-time continuous glucose monitoring users, and no relationship between sensor-based outcomes with the English index of multiple deprivation rank and deciles was found.

Empirical data from our group and others have therefore revealed that in the context of the lockdown period, glycaemic control in people with type 1 diabetes, albeit in the short term, was not significantly compromised and in most cases had improved. A plausible explanation is that the lockdown period provided an opportunity for people with type 1 diabetes to implement appropriate strategies, such as allowing time to bolus before meals and thinking about insulin dose adjustments according to activity levels, without being perturbed or distracted by other daily tasks or unpredictable factors at work. This alteration in behaviour during lockdown may have contributed to the increase in number of patients achieving the recommended sensor-based glycaemic targets [13]. In comparison, national data from the UK Freestyle Libre audit from November 2017 reported a median TIR of only $43 \%$ (IQR 27-56\%) [20]. Our data show that at least $30 \%$ more patients during lockdown were able to achieve clinically meaningful improvements to their TIR and sensor-based estimated HbA1c levels. Whether these are sustainable in the longer term and associated with reduced disease burden is at present unknown. Although an overall TIR improvement was observed, notably 71 patients (32\%) had significant TIR deterioration of $5 \%$ or more during both lockdown periods. Compared to those with improved TIR, these patients had higher baseline \% TIR and lower baseline $\mathrm{HbA} 1 \mathrm{c}$. Due to the retrospective nature of our study, no direct explanation can currently be provided. However, among the plausible reasons for this observation, based on anecdotal patient feedback during clinical consultations, include mental distress associated with loss of income and daily routine, reduced physical activity, increased snacking and weight gain. It is also unclear whether the impact of lockdown has a beneficial or detrimental impact on diabetes management in those without access to sensor-based technology or with type 2 diabetes, although emerging evidence currently suggests the latter [21, 22].

Mean $\mathrm{HbA1c}$ at baseline in our cohort was lower compared to the UK national diabetes audit data from England and Wales, which reported mean $\mathrm{HbA} 1 \mathrm{c}$ of $64 \mathrm{mmol} / \mathrm{mol}$ (8\%) for pump users and $71 \mathrm{mmol} / \mathrm{mol}(8.6 \%)$ for those on MDI [23]. This was accompanied by the relatively high median sensor use ( $>90 \%$ ) overall and scanning frequency in FSL users, which suggests that our cohort was motivated. Real-time CGM application in our clinical practice is in keeping with UK NICE recommendations which restrict its application to those with higher hypoglycaemia burden (significant impairment of hypoglycaemia awareness and/ or severe hypoglycaemia) [24]. CGM users in our analysis, however, had lower time below range at all 3 time points, 
highlighting the benefits and value of CGM hypoglycaemia alerts and notifications in this cohort.

To our knowledge, this is the first report from England to show the impact of lockdown measures on glycaemic outcomes in people with diabetes using glucose sensors in clinical practice. Ours is also the largest to date evaluating the impact of lockdown in both real-time (Dexcom G6) and flash glucose monitoring sensor (FSL) users on MDI and insulin pump therapy, thereby widening the scope of our results. Other strengths include the analysis pertaining to professionally recommended glycaemic targets and clinically meaningful improvement in glycaemic outcomes. We acknowledge the limitations of our study. Due to the retrospective nature of the analysis, information related to changes in insulin doses, frequency of contact and advice from healthcare professionals, eating and physical activity behaviours are not available and not collected. Therefore, the factors driving improvements in glycaemic outcomes remain speculative. Our findings were in those using glucose sensors and whose mean baseline $\mathrm{HbAlc}$ was lower than the UK national data, therefore limiting generalisability. We also excluded those whose glucose sensors were not linked to their smartphones, to ensure that only consistent data uploads during the lockdown period were available and used in our analysis.

In conclusion, we have shown that sensor-based glycaemic outcomes are improved during lockdown period in people with diabetes equipped with sensor glucose. Further work is required to evaluate its longer-term sustainability, and whether wider access to glucose sensors in the event of future lockdown measures may benefit those without access to this technology at present. This provides further opportunities for future diabetes service development during this global pandemic.

Acknowledgement The authors thank the patients and staff at Manchester Diabetes, Endocrine and Metabolism Centre.

Author contributions H.T and L.L conceived the idea for the analysis. H.T, J.N.P and L.L collected the data. L.L and H.T did or supported data analyses, including the statistical analyses. J.N.P, L.L, M.K.R, J.S, A.U and H.T interpreted the results. H.T and L.L wrote the manuscript. All authors critically reviewed the report. No writing assistance was provided. H.T and L.L had full access to all of the data in the study and take responsibility for the integrity of the data and the accuracy of the data analyses.

\section{Funding None.}

\section{Compliance with ethical standard}

Conflict of interest L.L. reports having received speaker honoraria from Animas, Abbott, Insulet, Medtronic, Novo Nordisk, Roche and Sanofi; having served on advisory panels for Animas, Abbott, Novo Nordisk, Dexcom, Medtronic, Sanofi and Roche; and having received research support from Novo Nordisk and Dexcom. MKR has received educational grant support from MSD and Novo Nordisk; has modest stock ownership in GSK; and has consulted for Roche. HT received research support from Dexcom. No other potential conflicts of interest relevant to this article were reported.

Ethical approval For this retrospective service evaluation study using routinely collected data, ethical approval is not requested. This service evaluation was registered with Manchester University NHS Foundation Trust (Reference Number: SE-013).

Informed consent For this type of study, formal consent is not required.

Open Access This article is licensed under a Creative Commons Attribution 4.0 International License, which permits use, sharing, adaptation, distribution and reproduction in any medium or format, as long as you give appropriate credit to the original author(s) and the source, provide a link to the Creative Commons licence, and indicate if changes were made. The images or other third party material in this article are included in the article's Creative Commons licence, unless indicated otherwise in a credit line to the material. If material is not included in the article's Creative Commons licence and your intended use is not permitted by statutory regulation or exceeds the permitted use, you will need to obtain permission directly from the copyright holder. To view a copy of this licence, visit http://creativecommons.org/licenses/by/4.0/.

\section{References}

1. DiMeglio LA, Evans-Molina C, Oram RA (2018) Type 1 diabetes, Lancet 391(10138):2449-2462. https://doi.org/10.1016/S0140 $-6736(18) 31320-5$

2. Chatterjee S, Davies MJ (2015) Current management of diabetes mellitus and future directions in care, Postgrad Med J 91(1081):612-621. https://doi.org/10.1136/postgradmedj-2014133200

3. McCarthy MM, Grey M (2018) Type 1 diabetes self-management from emerging adulthood through older adulthood, Diabetes Care 41(8):1608-1614. https://doi.org/10.2337/dc17-2597

4. van Duinkerken E, Snoek FJ, de Wit M (2019) The cognitive and psychological effects of living with type 1 diabetes: a narrative review, Diabet Med. https://doi.org/10.1111/dme.14216

5. Riazi A, Pickup J, Bradley C (2004) Daily stress and glycaemic control in type 1 diabetes: individual differences in magnitude, direction, and timing of stress-reactivity, Diabetes Res ClinPract 66(3):237-244. https://doi.org/10.1016/j.diabres.2004.04.001

6. Trento $\mathrm{M}$ et al (2009) Quality of life, coping ability, and metabolic control in patients with type 1 diabetes managed by group care and a carbohydrate counting program, Diabetes Care 32(11):e134. https://doi.org/10.2337/dc09-0903

7. Prime Minister's statement on coronavirus (COVID-19) 2020, https://www.gov.uk/government/speeches/pm-address-to-thenation-on-coronavirus-23-march-2020

8. Coronavirus Act 2020, https://www.legislation.gov.uk/ukpga /2020/7/contents/enacted, ed

9. Evans M, Welsh Z, Ells S, Seibold A (2020) The impact of flash glucose monitoring on glycaemic control as measured by HbA1c: a meta-analysis of clinical trials and real-world observational studies, Diabetes Ther 11(1):83-95. https://doi.org/10.1007/s1330 0-019-00720-0

10. Foster NC et al (2019) State of type 1 diabetes management and outcomes from the T1D exchange in 2016-2018, Diabetes TechnolTher 21(2):66-72. https://doi.org/10.1089/dia.2018.0384 
11. Aleppo G et al (2017) REPLACE-BG: a randomized trial comparing continuous glucose monitoring with and without routine blood glucose monitoring in adults with well-controlled type 1 diabetes, Diabetes Care 40(4):538-545. https://doi.org/10.2337/dc16-2482

12. Rodbard D (2017) Continuous glucose monitoring: a review of recent studies demonstrating improved glycemic outcomes, Diabetes TechnolTher 19(S3):S25-S37. https://doi.org/10.1089/ dia.2017.0035

13. Battelino $\mathrm{T}$ et al (2019) Clinical targets for continuous glucose monitoring data interpretation: recommendations from the international consensus on time in range, Diabetes Care 42(8):15931603. https://doi.org/10.2337/dci19-0028

14. Nathan DM et al (2008) Translating the A1C assay into estimated average glucose values, Diabetes Care 31(8):1473-1478. https:// doi.org/10.2337/dc08-0545

15. Andrikopoulos S, Johnson G (2020) The Australian response to the COVID-19 pandemic and diabetes-Lessons learned, Diabetes Res ClinPract 165:108246. https://doi.org/10.1016/j.diabr es.2020.108246

16. Verma A, Rajput R, Verma S, Balania VKB, Jangra B (2020) Impact of lockdown in COVID 19 on glycemic control in patients with type 1 diabetes mellitus, Diabetes Metab Syndr 14(5):12131216. https://doi.org/10.1016/j.dsx.2020.07.016

17. Bonora BM, Boscari F, Avogaro A, Bruttomesso D, Fadini GP (2020) Glycaemic control among people with type 1 diabetes during lockdown for the SARS-CoV-2 outbreak in Italy, Diabetes Ther. 11:1369-1379. https://doi.org/10.1007/s13300-020-00829 $-7$

18. Mesa A et al (2020) The impact of strict COVID-19 lockdown in Spain on glycemic profiles in patients with type 1 diabetes prone to hypoglycemia using standalone continuous glucose monitoring, Diabetes Res ClinPract 167:108354. https://doi.org/10.1016/j. diabres.2020.108354
19. Dover AR et al (2020) Assessment of the effect of the COVID19 lockdown on glycaemic control in people with type 1 diabetes using flash glucose monitoring, Diabet Med. https://doi. org/10.1111/dme.14374

20. Deshmukh H et al (2020) Effect of flash glucose monitoring on glycemic control, hypoglycemia, diabetes-related distress, and resource utilization in the Association of British Clinical Diabetologists (ABCD) nationwide audit, Diabetes Care. https://doi. org/10.2337/dc20-0738

21. Ghosh A, Arora B, Gupta R, Anoop S, Misra A (2020) Effects of nationwide lockdown during COVID-19 epidemic on lifestyle and other medical issues of patients with type 2 diabetes in North India, Diabetes Metab Syndr 14(5):917-920. https://doi. org/10.1016/j.dsx.2020.05.044

22. Ghosal S, Arora B, Dutta K, Ghosh A, Sinha B, Misra A (2020) Increase in the risk of type 2 diabetes during lockdown for the COVID19 pandemic in India: a cohort analysis, Diabetes Metab Syndr 14(5):949-952. https://doi.org/10.1016/j.dsx.2020.06.020

23. https://digital.nhs.uk/data-and-information/publications/statistica 1/national-diabetes-audit/report-1-care-processes-and-treatmenttargets-2017-18-full-report, ed

24. National Institute for Health and Care Excellence, type 1 diabetes in adults: diagnosis and management, https://www.nice.org.uk/ guidance/ng17

Publisher's Note Springer Nature remains neutral with regard to jurisdictional claims in published maps and institutional affiliations. 